IUCrJ

ISSN 2052-2525

BIOLOGY|MEDICINE

Received 29 October 2020

Accepted 7 April 2021

Edited by J. L. Smith, University of Michigan, USA

₹ These authors contributed equally to this work.

Keywords: Acinetobacter baumannii; cell-wall peptidoglycan biosynthesis; crystal structure; glycosyltransferases; MurG; superbugs.

PDB reference: MurG, $7 \mathrm{~d} 1 \mathrm{i}$

Supporting information: this article has supporting information at www.iucrj.org

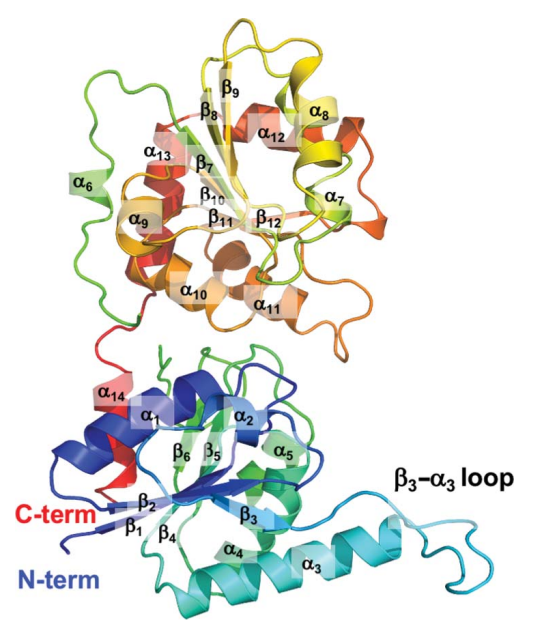

OPEN 2 ACCESS

\section{Putative hexameric glycosyltransferase functional unit revealed by the crystal structure of Acinetobacter baumannii MurG}

\author{
Kyoung Ho Jung, ${ }^{a} \neq$ Sunghark Kwon, ${ }^{b} \ddagger$ Chang Min Kim, ${ }^{a}$ Jun Hyuck Lee ${ }^{c, d *}$ and \\ Hyun Ho Park ${ }^{\mathrm{a} *}$
}

${ }^{\text {a} C o l l e g e ~ o f ~ P h a r m a c y, ~ C h u n g-A n g ~ U n i v e r s i t y, ~ S e o u l ~ 06974, ~ R e p u b l i c ~ o f ~ K o r e a, ~}{ }^{\mathbf{b}}$ Department of Biotechnology, Konkuk
University, Chungju, Chungbuk 27478, Republic of Korea, ' ${ }^{\mathbf{C}}$ Research Unit of Cryogenic Novel Material, Korea Polar
Research Institute, Incheon 21990, Republic of Korea, and ${ }^{\mathbf{d}}$ Department of Polar Sciences, University of Science and
Technology, Incheon 21990, Republic of Korea. ${ }^{*}$ Correspondence e-mail: junhyucklee@kopri.re.kr, xrayleox@cau.ac.kr

Lipid II, the main component of the bacterial cell wall, is synthesized by the addition of UDP- $N$-acetylglucosamine to the UDP- $N$-acetylmuramic acid pentapeptide catalyzed by the glycosyltransferase MurG. Owing to its critical role in cell-wall biosynthesis, MurG is considered to be an attractive target for antibacterial agents. Although the Mur family ligases have been extensively studied, the molecular mechanism of the oligomeric scaffolding assembly of MurG remains unclear. In this study, MurG from Acinetobacter baumannii (abMurG), a human pathogen, was characterized and its hexameric crystal structure was unveiled; this is the first homo-oligomeric structure to be described in the MurG family and the Mur family. Homogeneous protein samples were produced for structural studies using size-exclusion chromatography, the absolute molecular mass was calculated via multi-angle light scattering, and protein-protein interactions were analyzed using the PDBePISA server. abMurG was found to form homo-oligomeric complexes in solution, which might serve as functional units for the scaffolding activity of MurG. Furthermore, analysis of this structure revealed the molecular assembly mechanism of MurG. This structural and biochemical study elucidated the homo-oligomerization mechanism of MurG and suggests a new potential antibiotic target on MurG.

\section{Introduction}

Peptidoglycan, the major component of the bacterial cell wall, is a three-dimensional mesh structure formed by the polymerization of repeating disaccharide subunits cross-linked by short peptides (Vollmer \& Bertsche, 2008; Matteï et al., 2010). As peptidoglycan is essential for the growth and division of the cell wall of all eubacteria, the peptidoglycan-synthesis pathway is an attractive target for antibacterial agents (Chung et al., 2016; Basavannacharya et al., 2010; Kouidmi et al., 2014).

Peptidoglycan synthesis occurs in three different bacterial compartments: the cytoplasm, the membrane and the periplasm. During the cytoplasmic step, lipid II, the main peptidoglycan building unit, is produced by the action of the Mur family of enzymes (MurA-MurG; Smith, 2006; Miyachiro et al., 2019). Initially, MurA and MurB catalyze the generation of UDP- $N$-acetylmuramic acid (MurNAc) from UDP- $N$-acetylglucosamine (GlcNAc). Subsequently, a peptide moiety is assembled on UDP-MurNAc by the successive addition of L-alanine, D-glutamine, diaminopimelic acid or L-lysine, and dipeptide D-alanyl-D-alanine, generating the UDP-MurNAc pentapeptide. These additions are catalyzed by the Mur ligases MurC, MurD, MurE and MurF, respectively. 
In contrast, MurG belongs to the glycosyltransferases, which are one of the most diverse enzymatic groups. It binds to phospholipids on the cytoplasmic side of the membrane (Bupp \& Van Heijenoort, 1993). Further, the N-terminal hydrophobic patch of MurG is involved in membrane attachment (Ha et al., 2000). This enzyme catalyzes the addition of UDP-GlcNAc (another disaccharide subunit) to the UDP-MurNAc pentapeptide (called lipid I) at the end of the cytoplasmic stage, producing lipid II, which is then moved to the periplasm by flippases for further modification, polymerization and cross-linking to generate peptidoglycan [Fig. 1(a)] (Sham et al., 2014).
Considering that the enzymes involved in the peptidoglycan-biosynthesis pathway, including those belonging to the Mur family, are essential for cell viability, they are considered to be promising targets for antibacterial agents (Kouidmi et al., 2014). Although many clinically used antibiotics, such as penicillin derivatives ( $\beta$-lactams), vancomycin (glycopeptide) and cycloserine, target this peptidoglycan-biosynthesis pathway and have shown effective interference, no antibiotics targeting the Mur family are commercially available.

The need for the development of new antibiotic agents has recently been emphasized because of the emergence of superbugs, which are antibiotic-resistant bacteria that are

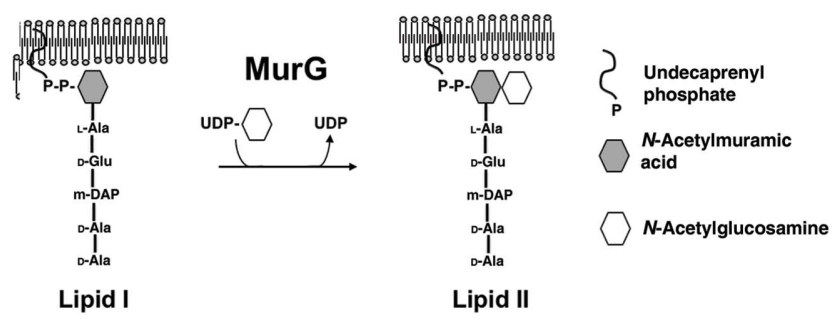

(a)

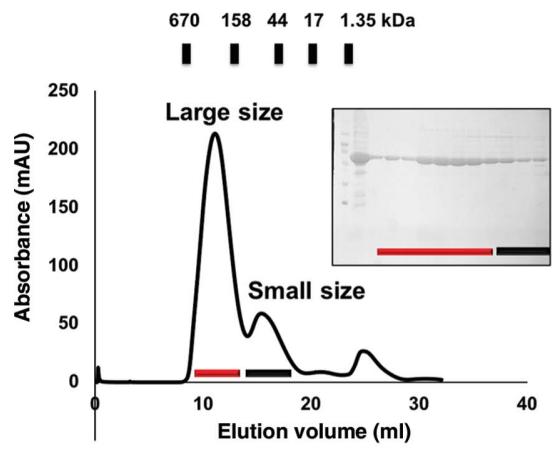

(b)

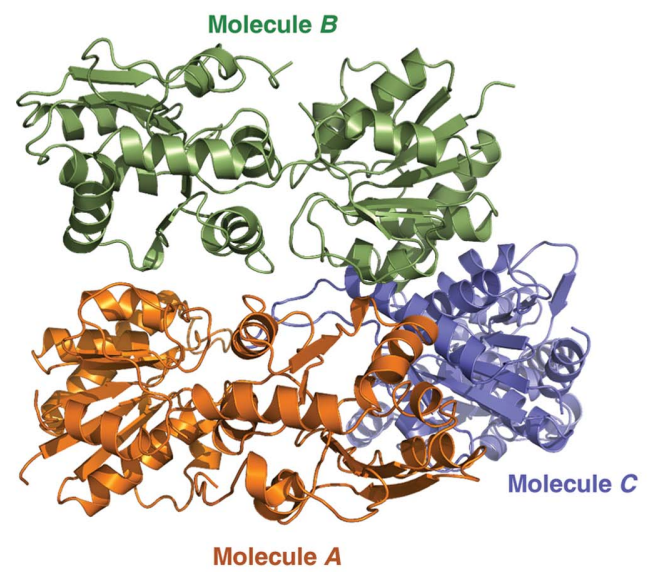

(f)

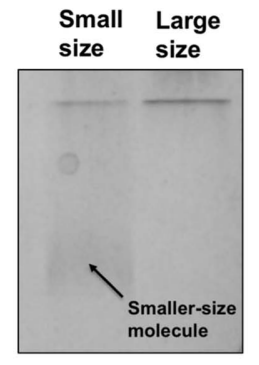

(c)

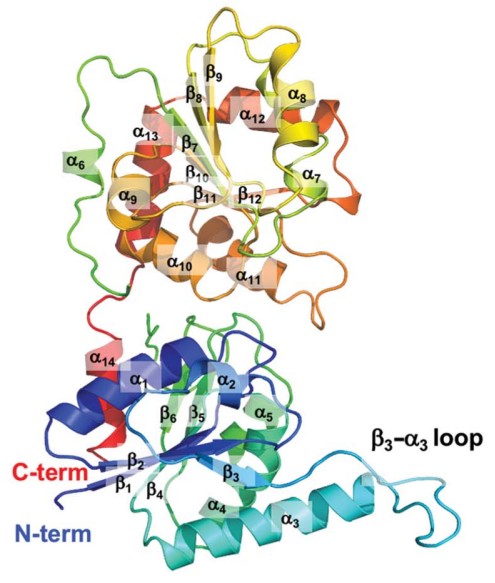

(d)

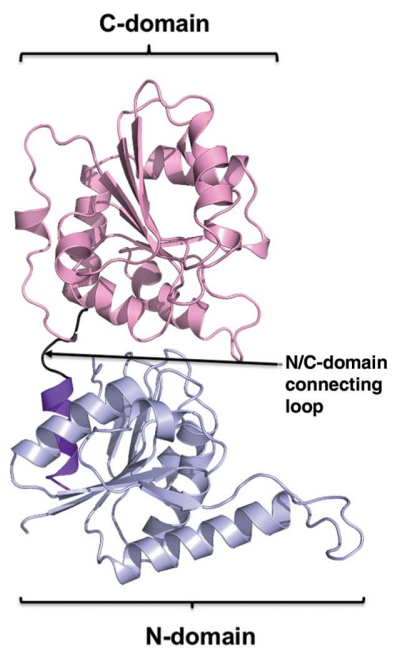

(e)

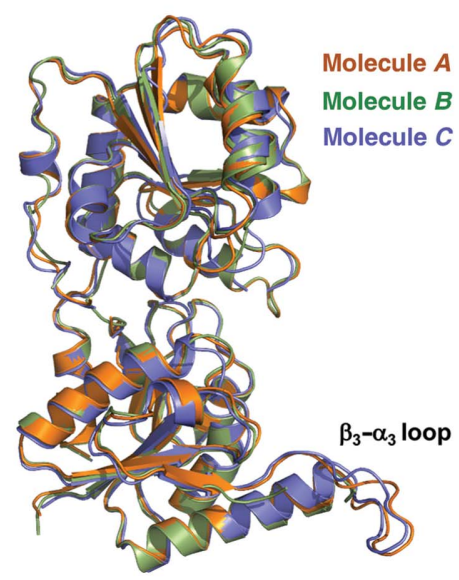

(g)

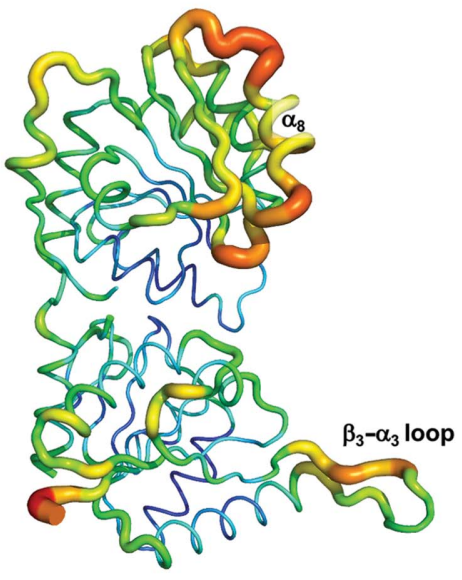

(h)

Figure 1

Crystal structure of abMurG. (a) Overview of the function of MurG. (b) Size-exclusion chromatography profile. Two peaks are labeled corresponding to an oligomer and a dimer. SDS-PAGE to assess the identity and purity is shown to the right of the two main peaks. The loaded fractions are indicated by black and red bars. $(c)$ Native PAGE gel. The loaded samples are indicated above the gel. $(d)$ Multi-colored cartoon representation of monomeric abMurG. The chain from the $\mathrm{N}$ - to $\mathrm{C}$-terminus is colored from blue to red. Helices and sheets are labeled $\alpha$ and $\beta$, respectively. (e) A cartoon representation of the structure of abMurG showing the domain boundary in the structure. $(f)$ Cartoon representation of the three abMurG molecules in an asymmetric unit. $(g)$ Superposition of the structures of the molecules found in one asymmetric unit. $(h)$ Putty representation showing the $B$-factor distribution. Rainbow colors from red to violet with increasing $B$-factor values were used for $B$-factor visualization. 
considered to be one of the greatest threats to global public health (Kumar, 2016; Burki, 2018). For example, Acinetobacter baumannii, a typical rod-shaped Gram-negative bacterium, is a human pathogen that causes hospital-derived infections and occasionally exhibits multiple drug resistance.

Owing to the critical activity of MurG in bacterial cell-wall synthesis and its attractiveness as an antibiotic target, structural and biochemical studies have been performed in order to understand its molecular mechanism (Ha et al., 1999; Mohammadi et al., 2007; van den Brink-van der Laan et al., 2003; Hu et al., 2004). Although previous studies of Escherichia coli MurG (ecMurG) have shown how MurG functions in the membrane and how its active site recognizes substrates (Ha et al., 2000; Hu et al., 2003), the molecular mechanism of the oligomeric scaffolding assembly of MurG, as identified in several bacterial species in recent studies (Laddomada et al., 2019), has remained unclear. This study characterized MurG from A. baumannii (abMurG). We revealed that abMurG forms a homo-oligomeric complex in solution that might be the functional unit for MurG scaffolding activity. We also determined the hexameric crystal structure of abMurG, which, to the best of our knowledge, is the first homo-oligomeric structure in the MurG family and even in the Mur family. Our structural study of hexameric MurG revealed the molecular assembly mechanism of MurG. These structural and biochemical studies are expected to shed light on the homooligomerization mechanism of MurG and to provide critical structural information for the design of drugs targeting MurG.

\section{Materials and methods}

\subsection{Protein expression and purification}

The expression plasmid for full-length abMurG corresponding to amino acids Met1-Met365 was constructed by inserting the synthesized gene product, digested at the NdeI and XhoI restriction sites, into pET-21a vector. The gene sequence was derived from GenBank (ID SVJ97884), and gene synthesis was conducted by BIONICS (Seoul, Republic of Korea). The expression vector containing the abMurG gene was delivered into the E. coli BL21 (DE3) strain by heat shock at $42^{\circ} \mathrm{C}$. The transformed bacteria were spread on a lysogeny broth agar plate containing kanamycin and incubated at $37^{\circ} \mathrm{C}$ for $16 \mathrm{~h}$. A single recombinant colony was selected and cultured overnight at $37^{\circ} \mathrm{C}$ in $5 \mathrm{ml}$ lysogeny broth containing $50 \mu \mathrm{g} \mathrm{ml}^{-1}$ kanamycin, following which the cells were transferred and cultured on a large scale (61). When the optical density at $600 \mathrm{~nm}$ reached approximately $0.6-0.7,0.5 \mathrm{mM}$ isopropyl $\beta$-D-1-thiogalactopyranoside was added to the medium to induce gene expression, and the cells were further cultured at $20^{\circ} \mathrm{C}$ for $18 \mathrm{~h}$ in a shaking incubator. Subsequently, the bacterial cells were harvested by centrifugation and the pellet was resuspended in $20 \mathrm{ml}$ lysis buffer $(20 \mathrm{~m} M$ Tris- $\mathrm{HCl}$ $\mathrm{pH} 7.9,500 \mathrm{~m} M \mathrm{NaCl}$ ). After adding phenylmethylsulfonyl fluoride, a serine protease inhibitor (Sigma-Aldrich, St Louis, USA), the cells were disrupted by sonication on ice with six bursts of $30 \mathrm{~s}$ each and a $60 \mathrm{~s}$ interval between bursts. The cell lysate was centrifuged at $10000 \mathrm{~g}$ and $4^{\circ} \mathrm{C}$ for $30 \mathrm{~min}$ to remove cell debris. The supernatant was collected and mixed with nickel-nitrilotriacetic acid resin solution (Qiagen, Hilden, Germany) by gentle agitation at $4^{\circ} \mathrm{C}$ overnight. The resulting mixture was inserted into a gravity-flow column preequilibrated with lysis buffer. The column was washed with $200 \mathrm{ml}$ washing buffer $(20 \mathrm{~m} M$ Tris- $\mathrm{HCl}$ pH 7.9, $500 \mathrm{~m} M$ $\mathrm{NaCl}, 25 \mathrm{~m} M$ imidazole) to remove unbound proteins. $3 \mathrm{ml}$ elution buffer $(20 \mathrm{~m} M$ Tris- $\mathrm{HCl}$ pH $7.9,500 \mathrm{~m} M \mathrm{NaCl}$, $250 \mathrm{~m} M$ imidazole) was then loaded into the column to elute the bound protein. The resulting eluate was concentrated to $20 \mathrm{mg} \mathrm{ml}^{-1}$ and was subsequently subjected to size-exclusion chromatography (SEC). SEC purification was conducted using an ÄKTAexplorer system (GE Healthcare, Chicago, USA) equipped with a Superdex 200 Increase 10/300 GL $24 \mathrm{ml}$ column (GE Healthcare) pre-equilibrated with SEC buffer $(20 \mathrm{~m} M$ Tris- $\mathrm{HCl} \mathrm{pH} 8.0,150 \mathrm{~m} M \mathrm{NaCl})$. The SEC peak fractions were pooled, concentrated to $8 \mathrm{mg} \mathrm{ml}^{-1}$, flashcooled in liquid $\mathrm{N}_{2}$ and stored at $-80^{\circ} \mathrm{C}$ until use. Protein purity was assessed by SDS-PAGE.

\subsection{SEC-MALS analysis}

The absolute molar mass of full-length abMurG in solution was determined using multi-angle light scattering (MALS). The target protein, purified by affinity chromatography using nickel-nitrilotriacetic acid resin, was filtered using a $0.2 \mu \mathrm{m}$ syringe filter and loaded onto a Superdex 200 10/300 gelfiltration column (GE Healthcare) that had been pre-equilibrated with SEC buffer. The mobile phase buffer was flowed at a rate of $0.4 \mathrm{ml} \mathrm{min}^{-1}$ at $25^{\circ} \mathrm{C}$. A DAWN TREOS MALS detector (Wyatt Technology, Santa Barbara, USA) was connected to the ÄKTAexplorer system (GE Healthcare). The molecular mass of bovine serum albumin was used as the reference value. The absolute molecular mass was assessed using ASTRA (Wyatt Technology).

\subsection{Native PAGE}

Different oligomeric states of abMurG in solution were assayed by native (nondenaturing) PAGE conducted on a PhastSystem (GE Healthcare) with pre-cast $8-25 \%$ acrylamide gradient gels (GE Healthcare). Coomassie Brilliant Blue was used for the staining and detection of bands. Different oligomeric states were evaluated based on the appearance of lower and upper bands on the native PAGE.

\subsection{Crystallization and data collection}

For initial crystal screening, $1 \mu 18 \mathrm{mg} \mathrm{ml}^{-1}$ protein solution in $20 \mathrm{~m} M$ Tris- $\mathrm{HCl} \mathrm{pH} \mathrm{8.0,150} \mathrm{mM} \mathrm{NaCl}$ was mixed with an equal volume of reservoir solution and the droplet was allowed to equilibrate against $500 \mu \mathrm{l}$ mother liquor using the hanging-drop vapor-diffusion method at $20^{\circ} \mathrm{C}$. Crystals were initially obtained using a buffer consisting of $0.1 \mathrm{M}$ Tris- $\mathrm{HCl}$ $\mathrm{pH}$ 8.0, $2.5 \mathrm{M} \mathrm{NaCl}, 0.2 \mathrm{M} \mathrm{MgCl}_{2}$. The crystallization conditions were further optimized and finally adjusted to a buffer composition of $0.1 M$ Tris- $\mathrm{HCl}$ pH 8.0, 2.6 M NaCl, $0.15 M$ calcium acetate. Diffraction-quality crystals appeared in three 
days and grew to maximum dimensions of $0.1 \times 0.1 \times 0.4 \mathrm{~mm}$. For data collection, the crystals were soaked in mother liquor supplemented with $30 \%(v / v)$ glycerol as a cryoprotectant, mounted and flash-cooled in an $\mathrm{N}_{2}$ stream at $-178^{\circ} \mathrm{C}$. The diffraction data were collected on the $5 \mathrm{C}$ beamline at the Pohang Accelerator Laboratory (PAL), Pohang, Republic of Korea at a wavelength of $0.9735 \AA$. The diffraction data were indexed, integrated and scaled using $H K L-2000$ (Otwinowski \& Minor, 1997).

\subsection{Structure determination and analysis}

The abMurG structure was determined by molecular replacement using Phaser (McCoy, 2007). The ecMurG structure (PDB entry 1f0k; Ha et al., 2000), which has 43\% amino-acid sequence homology to abMurG, was used as the search model. The initial model was built automatically with AutoBuild in Phenix (Liebschner et al., 2019) and was completed with Coot (Emsley et al., 2010). Model refinement was iteratively performed using phenix.refine in Phenix. The quality of the model was validated using MolProbity (Chen $e t$ al., 2010). All structural figures were generated using PyMOL (DeLano \& Lam, 2005).

\subsection{Mutagenesis}

Site-directed mutagenesis was conducted using a QuikChange kit (Stratagene) according to the manufacturer's protocols. Mutagenesis was then confirmed by sequencing. Mutant proteins were prepared using the method described above.

\subsection{Sequence alignment}

The amino-acid sequences of MurG from various species were analyzed using Clustal Omega (http://www.ebi.ac.uk/ Tools/msa/clustalo/).

\subsection{Accession code}

Coordinates and structure factors have been deposited in the RCSB Protein Data Bank with PDB code 7d1i.

\section{Results and discussion}

\subsection{Overall structure of abMurG}

To explore the structure of abMurG, the full-length MurG cDNA from A. baumannii, coding for a protein of 365 amino acids, was synthesized and cloned into pET-21a expression vector. To produce homogeneous protein samples for structural studies, we conducted quick two-step chromatography: affinity chromatography followed by SEC. This purification process generated two homogeneous protein samples corresponding to the large-size and small-size peaks, which were used for crystallization [Fig. 1(b)]. Only protein sample from the large-size peak was successfully crystallized. On the native PAGE, a smear of putative smaller-sized molecules of MurG was detected, indicating that MurG forms both large and small oligomeric molecules in solution [Fig. 1(c)]. Finally, a $3.49 \AA$
Table 1

Data-collection and refinement statistics.

Values in parentheses are for the outermost resolution shell.

\begin{tabular}{ll}
\hline Data collection & \\
Space group & $P 4_{3} 2.2$ \\
$a, b, c(\AA)$ & $182.91,182.91,156.55$ \\
$\alpha, \beta, \gamma\left({ }^{\circ}\right)$ & $90,90,90$ \\
Resolution range $(\AA)$ & $50.00-3.49$ \\
Total reflections & 257452 \\
Unique reflections & 31262 \\
Multiplicity & $8.2(3.4)$ \\
Completeness $(\%)$ & $90.8(82.1)$ \\
Mean $I / \sigma(I)$ & $10.2(2.1)$ \\
$R_{\text {merge }}(\%)$ & $6.1(44.1)$ \\
Wilson $B$ factor $(\AA)$ & 71.04 \\
Refinement & \\
Resolution range $(\AA)$ & $48.25-3.49$ \\
Reflections & 31201 \\
$R_{\text {work }}(\%)$ & $22.28(28.13)$ \\
$R_{\text {free }}(\%)$ & $27.16(33.28)$ \\
No. of molecules in the asymmetric unit & 3 \\
No. of non-H atoms & 7706 \\
Average $B$-factor values $(\AA)$ & \\
Molecule $A$ & 70.84 \\
Molecule $B$ & 67.66 \\
Molecule $C$ & 66.94 \\
Ramachandran plot & \\
Favored $(\%)$ & 96.42 \\
Allowed $(\%)$ & 3.58 \\
Outliers $(\%)$ & 0 \\
Rotamer outliers $(\%)$ & 4.2 \\
Clashscore & 6.23 \\
Root-mean-square deviations & \\
Bonds $(\AA)$ & 0.008 \\
Angles $\left({ }^{\circ}\right)$ & 0.928 \\
\hline & \\
\hline &
\end{tabular}

$\dagger R_{\text {merge }}=\sum_{h k l} \sum_{i}\left|I_{i}(h k l)-\langle I(h k l)\rangle\right| / \sum_{h k l} \sum_{i} I_{i}(h k l)$, where $I(h k l)$ is the observed intensity of reflection $h k l$ and $\langle I(h k l)\rangle$ is the average intensity obtained from multiple measurements.

resolution crystal structure of abMurG was solved and refined to $R_{\text {work }}=22.28 \%$ and $R_{\text {free }}=27.16 \%$. The crystallographic and refinement statistics are summarized in Table 1.

The crystal structure of abMurG showed the typical fold of MurG, containing two distinct domains (N- and C-domains) [Figs. $1(d)$ and $1(e)$ ]. It was composed of $14 \alpha$-helices and 12 $\beta$-sheets, and there was an extraordinarily long loop between $\beta 3$ and $\alpha 3$ (hereafter named the $\beta 3-\alpha 3$ loop) [Fig. 1(d)]. The two distinctly separated $\mathrm{N}$ - and $\mathrm{C}$-domains, which exhibit an $\alpha / \beta$ open-sheet structure, were connected by an N/C-domain connecting loop [Fig. 1(e)]. The $\mathrm{N}$-domain consisted of six $\alpha$-helices $(\alpha 1-\alpha 5$ and $\alpha 14)$ and six $\beta$-sheets $(\beta 1-\beta 6)$, whereas the $\mathrm{C}$-domain consisted of eight $\alpha$-helices $(\alpha 6-\alpha 12)$ and six $\beta$-sheets $(\beta 7-\beta 12)$ [Figs. $1(d)$ and $1(e)$ ]. The last helix at the C-terminus, $\alpha 14$, was located in the $\mathrm{N}$-domain; it was involved in forming the $\alpha / \beta$ open-sheet structure of the $\mathrm{N}$-domain [Figs. $1(d)$ and $1(e)$ ]. Three molecules were found in the asymmetric unit: $A, B$ and $C$ [Fig. 1(f)]. Models of each molecule were constructed and included residues 8-365 for molecules $A$ and $B$, and residues 9-365 for molecule $C$. Seven residues from the $\mathrm{N}$-terminus and several loops were not included in the model due to poor electron density. The final model contained residues 8-166 and 175-365 for molecule $A$, residues 8-66, 77-168 and 175-365 for molecule $B$, and residues 9-166, 173-295 and 299-365 for molecule $C$ [Fig. 1( $f)]$. In the asymmetric unit, molecule $B$ was stacked on molecule $A$, 
and molecule $C$ was on the right side of molecule $A$ [Fig. 1 $(f)]$. The structures of the three molecules in the same asymmetric were nearly identical, with root-mean-square deviations of 0.65-0.87 $\AA$ over $338 \mathrm{C}^{\alpha}$ atoms [Fig. $1(\mathrm{~g})$ ]. Despite this structural similarity, structural discrepancy was detected in the region of the $\beta 3-\alpha 3$ loop. The position of this loop in molecule $A$ was not identical to that in molecule $C$. In molecule $B$, this loop was not included in the final model due to untraceable electron density, indicating that this region might be flexible. $B$-factor analysis showed that the structure of abMurG contained two high $B$-factor regions, including the $\beta 3-\alpha 3$ loop and $\alpha 8$ and its connecting loops [Fig. 1(h)], which supported our hypothesis that the $\beta 3-\alpha 3$ loop is a flexible region in the structure of abMurG.

\section{2. abMurG forms hexameric homo-oligomeric complex structures}

Although structural studies have provided no direct evidence, a highly oligomeric state of MurG has consistently been proposed as a functional unit by biochemical, biophysical and cellular studies (Laddomada et al., 2019; Ha et al., 1999).
As we solved the structure of abMurG from a highly oligomeric protein sample, we analyzed the crystallographic packing to search for symmetric molecules and found three molecules $\left(A^{\prime}, B^{\prime}\right.$ and $\left.C^{\prime}\right)$ that formed a hexameric structure with the three molecules found in the asymmetric unit [Fig. 2(a)]. This hexamer was formed by two trimeric molecules stacked against each other, forming a two-layer structure with threefold symmetry [Figs. 2(b) and 2(c)]. Molecules $A^{\prime}, B$ and $C^{\prime}$ were located in the top layer and molecules $A, B^{\prime}$ and $C$ in the bottom layer [Fig. 2(b)]. The top view of the complex shows that the two trimeric layers did not completely overlap [Fig. 2(c)]. The bottom layer flipped, rotated approximately $30^{\circ}$ and stacked on the bottom layer, indicating that the hexamer of abMurG is constructed of six identical subunits that are arranged as a trimer of asymmetric dimers with $D 3$ (or 32) symmetry [Figs. 2(b) and 2(c)]. When the trimeric form of abMurG was considered as the basic unit for the formation of this hexameric complex, abMurG was first trimerized and two trimers then interacted to form the hexameric structure. Another possibility for hexamer formation is that the abMurG dimer might be a protomer of this hexameric complex, and the dimers further interact to extend and form the hexameric

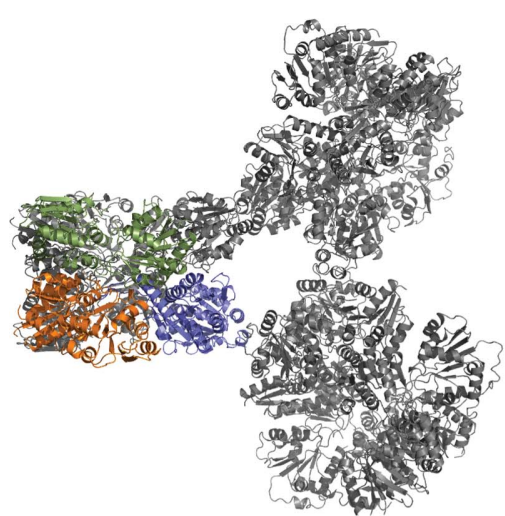

(a)

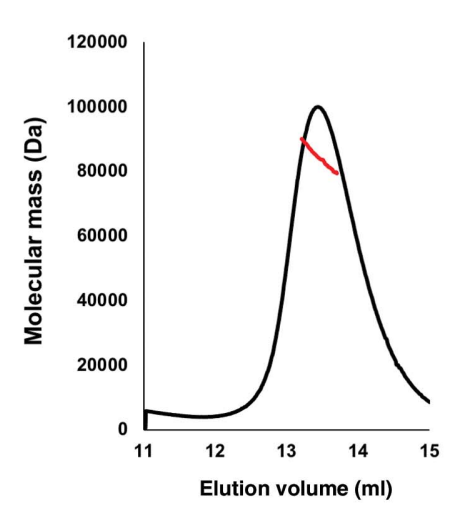

(d)

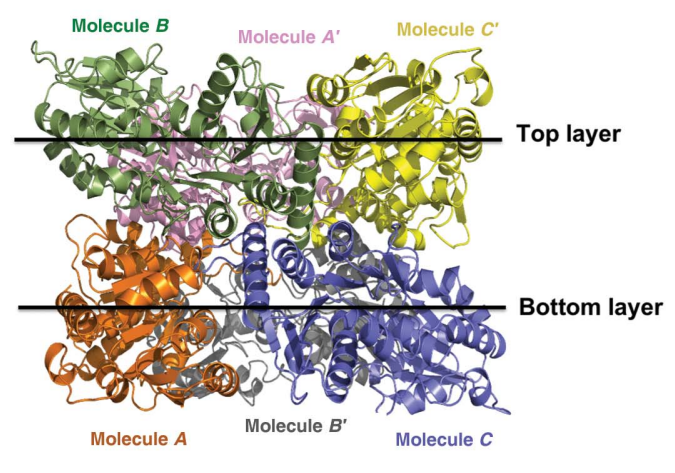

(b)

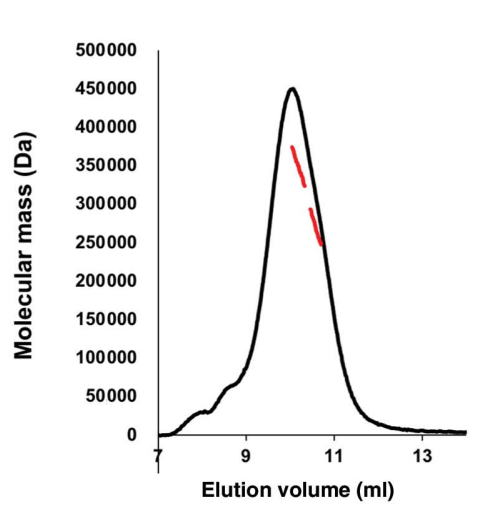

(e)

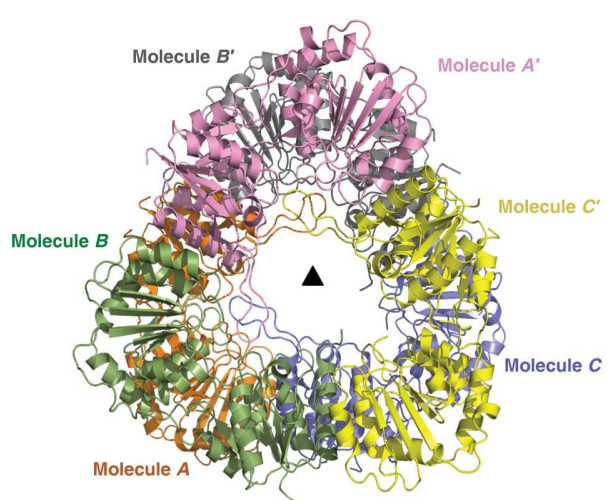

(c)

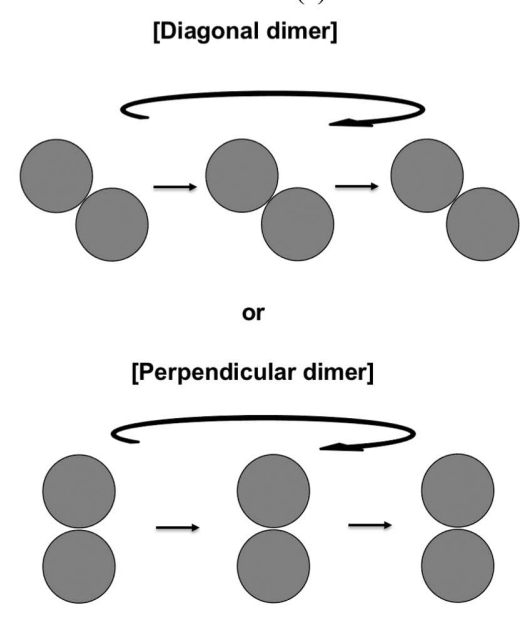

$(f)$

Figure 2

Hexameric structure of abMurG. (a) Crystallographic packing symmetry analysis. Three abMurG molecules in the asymmetric unit are indicated by color cartoons, whereas other symmetric molecules are indicated by gray ribbon structures. $(b, c)$ Tentative hexameric structure of abMurG generated by the symmetry analysis: side $(b)$ and top $(c)$ views. $(d, e)$ Multi-angle light scattering (MALS) profiles derived from the first $(d)$ and second $(e)$ size-exclusion chromatography peaks. The red line indicates the experimental molecular mass analyzed by MALS. $(f)$ Schematic planar diagram showing the hexamer assembly strategy of two tentative dimers. 
complex. In this case, the dimerized protomers of abMurG could be either diagonal or perpendicular dimers. To understand complex formation, we analyzed the exact stoichiometry of abMurG in solution by calculating the absolute molecular mass using MALS. We performed MALS experiments using the highly oligomeric and dimeric peaks obtained in SEC. The experimental molecular mass of the dimer-sized peak was $79.3 \mathrm{kDa}(1.1 \%$ fitting error) [Fig. 2(d)]. The theoretical molecular weight of abMurG, including the C-terminal His tag, is $39.4 \mathrm{kDa}$; hence, this experimental value confirms that abMurG is a dimer in this peak. In contrast, the molecular mass of the highly oligomeric peak was $280.5 \mathrm{kDa}(4.8 \%$ fitting error), confirming that abMurG is a hexamer in this peak [Fig. 2(e)]. Based on these results, we concluded that abMurG forms dimers, and not trimers, in solution, and that these dimers can further assemble into a highly oligomeric form; in this case, a hexamer. The dimeric central building block might be formed by diagonal or perpendicular dimers
[Fig. 2(f)]. As both dimers might be formed in solution, furthur studies are needed to fully understand the assembly mechanism of hexameric abMurG.

To understand the assembly details of hexameric abMurG, we analyzed protein-protein interactions (PPIs) using PDBePISA (Krissinel \& Henrick, 2007). According to the PPI computations, a hexameric quaternary structure was suggested as a stable form for abMurG in solution. The buried surface of the trimeric complex represented by the asymmetric unit was $5138 \AA^{2}$ of the total accessible surface area of $44718 \AA^{2}$, which indicated that $11.4 \%$ of the surface was buried on the formation of a trimeric complex. When abMurG formed a hexameric complex, the total hexameric surface was $81159 \AA^{2}$ and the surface buried on formation of the hexameric complex was $18554 \AA^{2}$, representing $41.4 \%$ of the total surface area. Further analysis indicated that hexameric abMurG was formed using three different interactions (types 1,2 and 3) [Fig. 3(a)]. If the three molecules $(A, B$ and $C)$ represented by

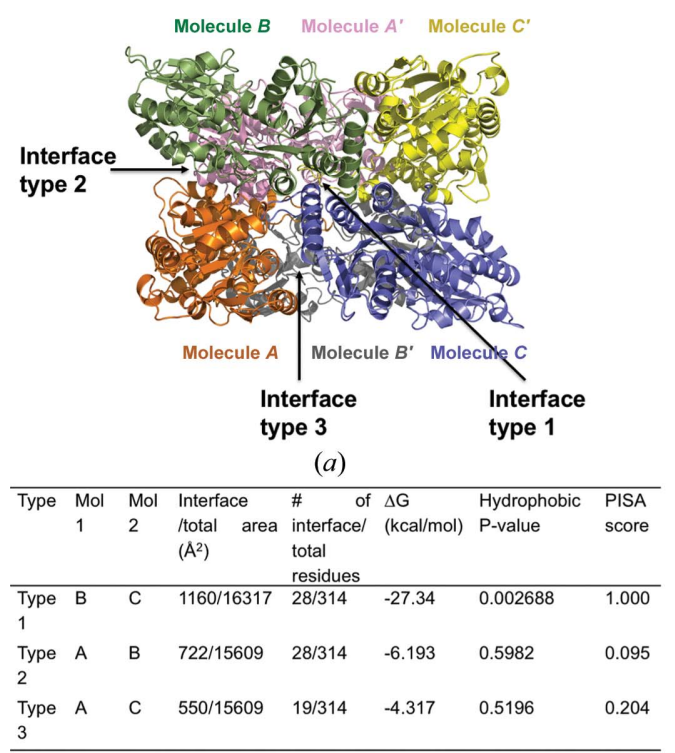

(b)

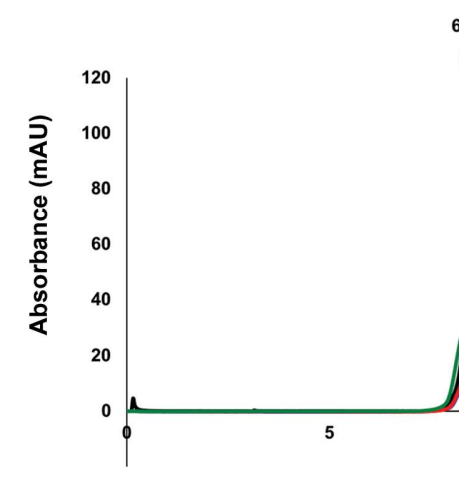

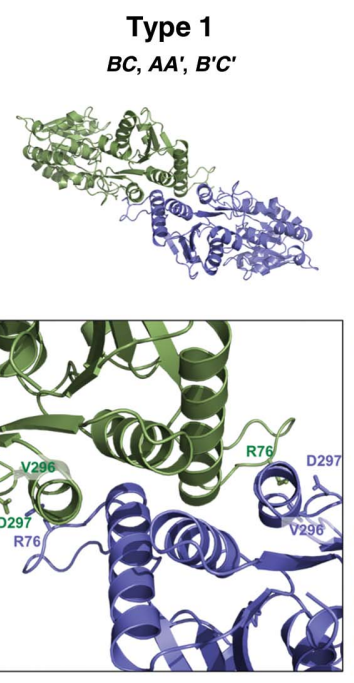

(c)

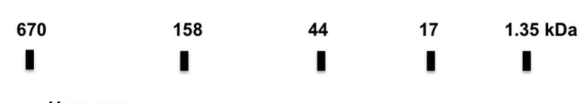

Hexamer

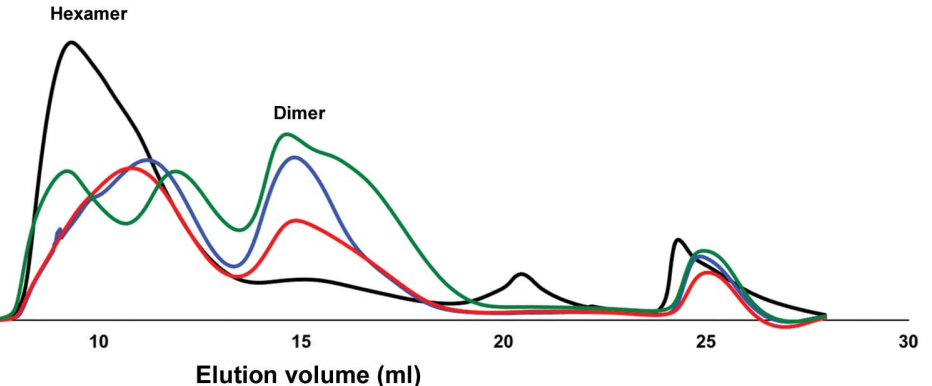

$(f)$
Type 2 $A B, A^{\prime} B$ ', $C C^{\prime}$
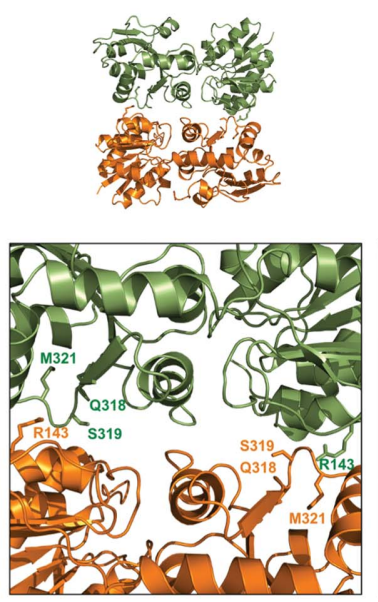

(d)
Type 3 $A C, A B^{\prime}, B^{\prime} C, B C^{\prime}, A^{\prime} B, A^{\prime} C^{\prime}$
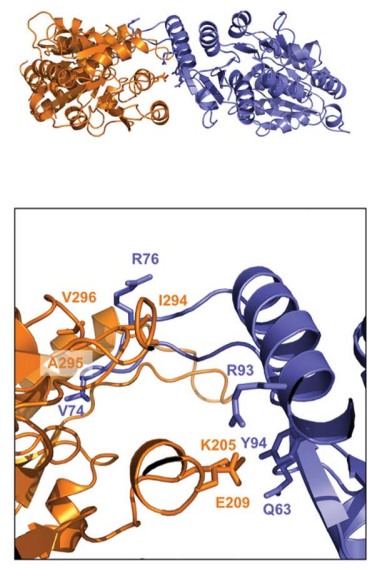

(e)

Figure 3

Details of the interface formed by the hexameric homo-oligomeric complex of abMurG. (a) Cartoon representation of the hexameric abMurG complex. Three different types of interfaces (types 1-3) formed by the hexameric complex are indicated by black arrows. (b) Table summarizing the interaction details of each type of interface. $(c, d, e)$ Cartoon representation of each type of interaction interface: type $1(c)$, type $2(d)$ and type $3(e)$. The position of each interface and the molecules involved in the formation of each interface are shown in the upper panel. Close-up views of each interface showing the residues involved in the formation of the interface are provided in the lower panels. $(f)$ Verification of the interfaces by mutagenesis. Size-exclusion chromatography profiles comparing the positions of eluted peaks between wild-type abMurG and various mutants with disrupted type 1 (R76D), type 2 (S319K) and type 3 (E209R) interfaces. 
the asymmetric unit are considered as building blocks for the hexameric structure, molecules $B$ and $C$ have type 1 interactions, molecules $A$ and $B$ have type 2 interactions, and molecules $A$ and $C$ have type 3 interactions [Figs. 3(a) and $3(b)]$. According to the PDBePISA analysis, the interface of type 1 inter-

Table 2

Structural similarity search using $D A L I$.

\begin{tabular}{lllll}
\hline Protein (PDB code) & Z-score & R.m.s.d. $(\AA)$ & Identity (\%) & Reference \\
\hline MurG from E. coli (1f0k) & 42.9 & 1.5 over 331 C $\mathrm{C}^{\alpha}$ & 43 & Ha et al. $(2000)$ \\
MurG-UDP-GlcNAc from E. coli $(1 \mathrm{nlm})$ & 41.5 & 2.0 over 329 C & 43 & Hu et al. $(2003)$ \\
MGD1 from A. thaliana (4wyi) & 23.7 & 3.3 over 285 C $\mathrm{C}^{\alpha}$ & 19 & Rocha et al. $(2016)$ \\
KCN28 from Kitasatospora $(6 \mathrm{j} 31)$ & 23.3 & 3.6 over 297 $\mathrm{C}^{\alpha}$ & 18 & Shi et al. $(2019)$ \\
\hline
\end{tabular}
actions had a complex-formation significance score of 1.000 (the score ranges from 0 to 1 as the relevance of the interface to complex formation increases), whereas the interfaces of type 2 and type 3 interactions had scores of 0.095 and 0.204 , respectively [Fig. 3(b)]. These results imply that type 1 interactions are the most significant interaction force in forming the hexameric abMurG complex.

A total of 12 interfaces are formed using the three different types of interactions in hexameric abMurG: the interactions between molecules $B$ and $C$, molecules $A$ and $A^{\prime}$, and molecules $B^{\prime}$ and $C^{\prime}$ are type 1 interactions [Fig. 3(c)], those between molecules $A$ and $B$, molecules $A^{\prime}$ and $B^{\prime}$, and molecules $C$ and $C^{\prime}$ are type 2 interactions [Fig. 3(d)], and six interfaces, between molecules $A$ and $C, A$ and $B^{\prime}, B^{\prime}$ and $C, B$ and $C^{\prime}, A^{\prime}$ and $B$, and $A^{\prime}$ and $C^{\prime}$, are formed by type 3 interactions [Fig. 3(e)]. Type 1 interactions mainly involve hydrogen bonds and salt bridges formed by the side chains of Arg76 and Asp297 and the main chain of Val296 [Fig. 3(c)]. Arg76 of one molecule forms salt bridges with Asp297 of the other molecule; Arg76 also forms hydrogen bonds to the main chain of Val296. In type 2 interactions, Arg143 forms hydrogen bonds to Gln318, Ser319 and Met321 from the neighboring molecule [Fig. 3(d)]. In the type 3 interface, Gln63, Val74, Arg76, Arg93 and Tyr94 of one molecule interact with Lys205, Glu209, Ile294, Ala295 and Val296 of the opposite molecule [Fig. 3(e)]. A structure-based mutagenesis study confirmed the analyzed interface. Arg76, Ser329 and Glu209 were analyzed as the main interface residues in type 1, type 2 and type 3 interactions, respectively. They were mutated to aspartic acid, lysine and arginine, producing the R76D, S319K and E209R mutants, respectively. Each mutant was purified, and the effects of the mutation in disruption of the hexameric complex were analyzed using SEC. The same protein concentration $\left(\sim 2 \mathrm{mg} \mathrm{ml}^{-1}\right.$ ) was used in the SEC experiments to compare the peak sizes. As indicated in Fig. 3(f), all three mutants had a definite disruptive effect on the hexameric complex, producing a higher dimeric peak than that produced by the wild type in the SEC profile. This indicates that abMurG forms a putative hexameric complex in solution, the assembly of which is mediated by the three types of interactions analyzed in this structural study. Notably, the S319K mutant produced one additional peak between the dimer and hexamer, implying that disruption of the type 2 interface by S319K mutagenesis produced new oligomeric forms.

\subsection{Comparison of the abMurG and ecMurG structures}

To find evidence to infer the molecular mechanism underlying the hexameric assembly of abMurG and its functional

role, we investigated its structural homologs using the $D A L I$ server (Holm \& Sander, 1995) and compared each of these structures with that of abMurG. This investigation revealed two structures of ecMurG, a substrate-free form (PDB entry 1 f0k; Ha et al., 2000) and a UDP-GlcNAc-bound form (PDB entry 1nlm; Hu et al., 2003), as the most structurally similar proteins (Table 2). Considering that most bacteria contain the MurG enzyme, it is noteworthy that ecMurG is the only structure reported to date; this might be because MurG is located on the membrane (van der Brink-van der Laan et al., 2003), making its structural study challenging to perform because of solubility issues. Two other structures, MGD1 from Arabidopsis (PDB entry 4wyi; Rocha et al., 2016) and KCN28 from Kitasatospora (PDB entry 6j31; Shi et al., 2019), which are unrelated to MurG (with low sequence homology), were found as the third and fourth matches, respectively (Table 2). The sequence homology between abMurG and ecMurG was around $43 \%$. ecMurG is the most enzymatically, biochemically and structurally studied MurG enzyme [Fig. 4(a)] (Men et al., 1998; Ha et al., 1999; Mohammadi et al., 2007). Although previous studies indicate that E. coli and Bordetella pertussis MurG form an oligomeric scaffold during cell-wall synthesis (Laddomada et al., 2019; Ha et al., 1999), the structure of ecMurG has been found to be a monomer in the presence and absence of substrate (Ha et al., 2000; Hu et al., 2003). Structural comparison by the superposition of monomeric abMurG with monomeric ecMurG showed that the overall fold was the same, with a root-mean-square deviation of $1.5 \AA$. However, the positions and lengths of several loops in abMurG differed from those of the equivalent loops in ecMurG [Fig. 4(b)]. Nevertheless, the structures of the $\alpha / \beta / \alpha$ motif and the GGS loop, which are essential for donor-substrate (UDP-GlcNAc) binding, and the HEQN loop, which is critical for accommodation of the acceptor substrate (lipid I), were conserved [Fig. 4(b)]. The sequences of these regions were also conserved among various species [Figs. 4(a) and 4(c)]. However, there were also regions with structural discrepancy. In particular, the $\beta 3-\alpha 3$ loop of abMurG, which was predicted to be a flexible region, was in an extended form, whereas the $\beta 3-\alpha 3$ loop of ecMurG was bent towards the HEQN loop [Figs. 4(b) and $4(d)$ ]. This bending might be a critical structural transition to fix the acceptor substrate during enzymatic activity of MurG because the $\beta 3-\alpha 3$ loop approaches the acceptor-binding site [Fig. 4(d)]. However, the bent $\beta 3-\alpha 3$ loop in ecMurG was a common structural feature in the presence and absence of substrate [Fig. 4(b)] (Hu et al., 2003; Ha et al., 2000), and is possibly a structural feature of MurGs from several species, including E. coli. Structural comparison of the active site of 
abMurG with that of ecMurG showed that most residues around the active site were sequentially and structurally conserved. However, the precise locations of several residues, including Gly199, Gly200, Ser201 and Phe254, were not identical [Figs. 4(e) and 4(f)].

As it is known that ecMurG also forms a highly oligomeric complex in solution (Ha et al., 1999), hexameric ecMurG was modeled using the hexameric abMurG structure as a template [Figs. $4(g)$ and $4(h)$ ]. Because our abMurG structure indicated that the $\beta 3-\alpha 3$ loop was involved in type 1 interactions and further assembly of the abMurG hexameric complex, we analyzed the role of this region of ecMurG in the proposed hexameric ecMurG complex. Analysis of the bent $\beta 3-\alpha 3$ loop region in the hexameric structure of ecMurG showed that type 1 interactions failed because of a clash of the two molecules involved in these interactions, indicating that this bent loop inhibits MurG assembly [Fig. 4(i)].

\subsection{Tentative working model of hexameric MurG on the membrane}

Based on previous reports and the findings of the current structural study, we concluded that MurG forms a putative hexameric complex, the assembly of which may be speciesdependent. However, structural transition of the $\beta 3-\alpha 3$ loop from the extended to the bent form might be critical for

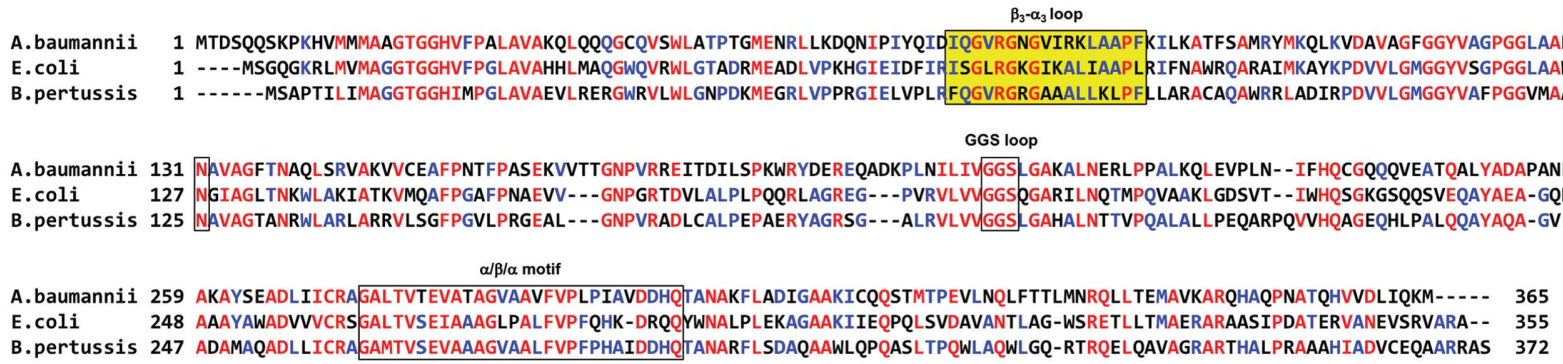

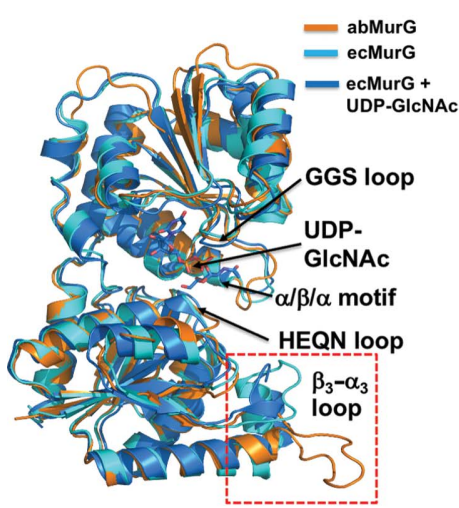

(b)

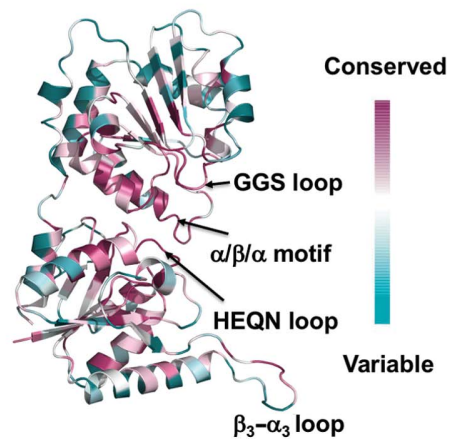

(c)

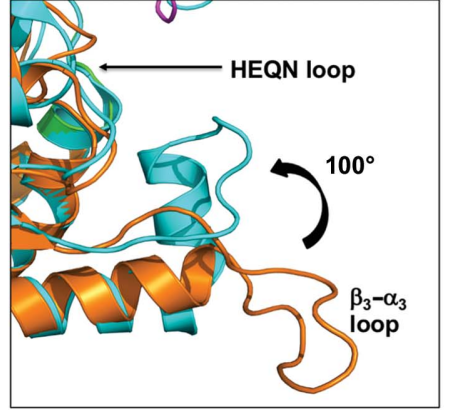

(d)

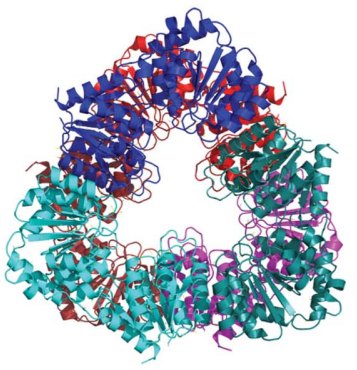

(g)

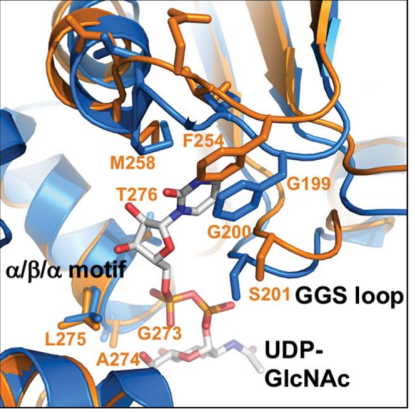

(e)

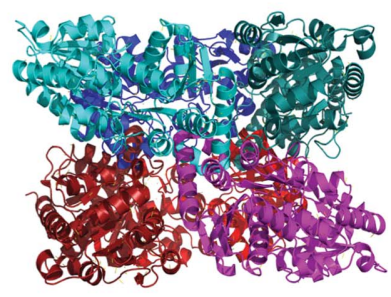

(h)

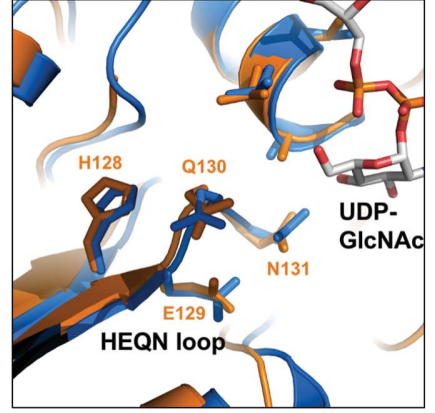

(f)

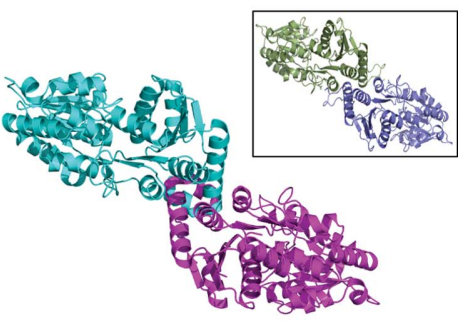

(i)

Figure 4

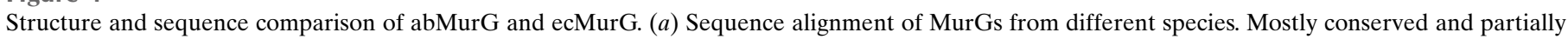

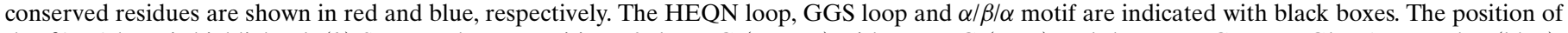

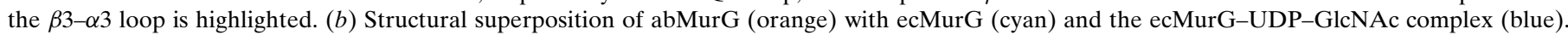

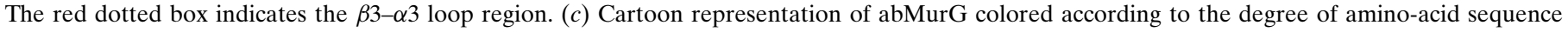

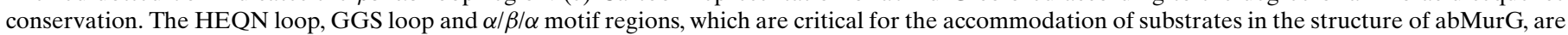

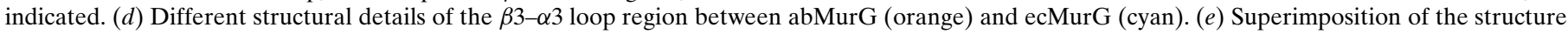

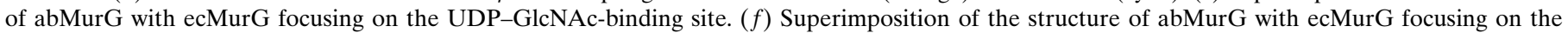

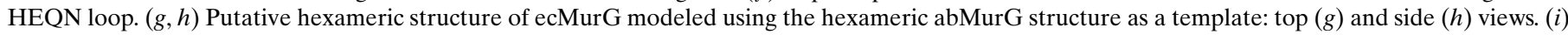

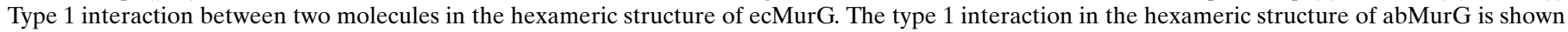
in a black box for comparison. 
regulation of the hexameric complex in the hexamer-mediated scaffolding function of MurG, and this process might be common to all MurG enzymes. Because MurG is located on the membrane, it has been questioned how hexameric MurG can work on the membrane. In addition, a tentative model describing how a hexameric MurG would work with dimeric MraY and flippase components for feeding the substrate and flipping the product during lipid II formation, respectively, would be noteworthy. However, due to an absence of information on the membrane anchoring of hexameric MurG, it might be difficult to speculate on the membrane attachment of MurG to work on the lipidated substrate and its cooperativity with MraY and flippase on the membrane. To speculate on the tentative membrane-docking region in MurG, we used the Membrane Protein Interface Recognition (MODA) server (Kufareva et al., 2014). According to the calculations by this server, two regions were picked as tentative membranedocking regions [Fig. 5(a)]. One region was the $\beta 3-\alpha 3$ loop part, which is critical for hexamer assembly. The other region was a small helix connected by a loop located on the surface of the MurG monomer [Fig. 5(a)]. This region was also exposed to the outside in hexameric MurG [Fig. 5(b)]. If the $\beta 3-\alpha 3$ loop part is involved in hexamer formation, as analyzed in the current hexameric structure, the small helix connected by a loop located on the surface of MurG might be a candidate region for membrane anchoring. Based on this observation, we speculated on the working model of hexameric MurG in the intracellular part of the membrane [Fig. 5(c)]. MurG might wrap up the lipid I substrate on the membrane by forming a hexamer and anchoring to the membrane. In this state, $\mathrm{N}$-acetylglucosamine (another substrate) might access the active site of hexameric MurG through the hole formed by subunits that are located on the opposite sides of membraneanchoring subunits. Although a model has been proposed, the precise membrane-anchoring process and cooperativity mechanism of hexameric MurG with MraY and flippase need to be analyzed by either a structural study or a biochemical study.

Currently, the application of newly discovered MurG inhibitors as next-generation antibiotics stems from competitive substrate analogs, which are either UDP-GlcNAc-mimicking or lipid I-mimicking compounds. However, to date no successful results have been reported. If MurG oligomerization, as introduced in this study, is critical for the activity of this enzyme, targeting the interface might be an alternative approach. Overall, understanding the assembly mechanism

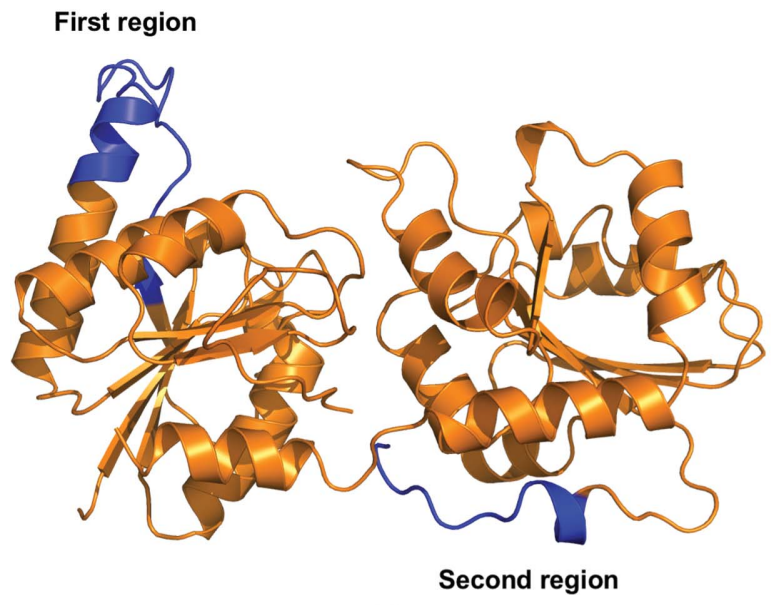

(a)

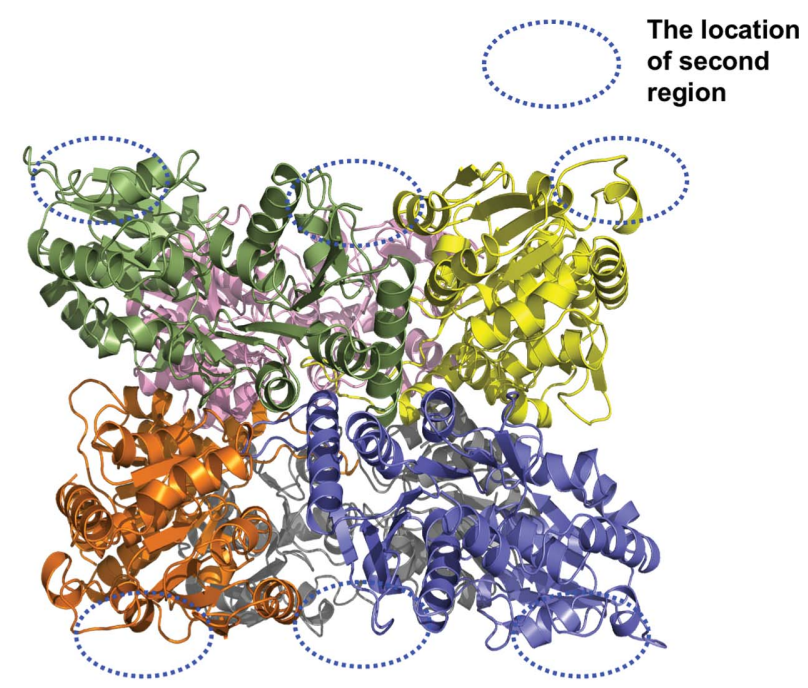

(b)
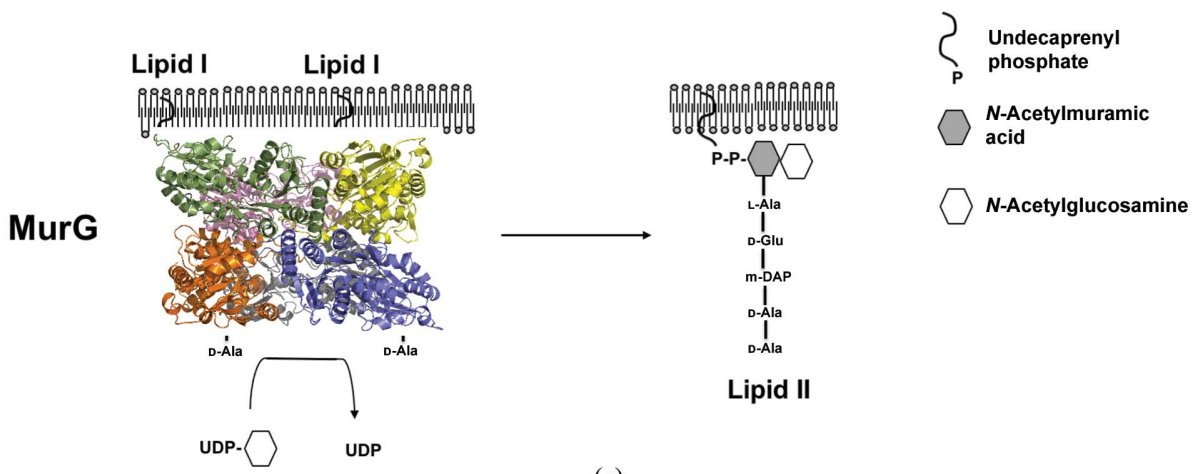

(c)

Figure 5

Proposed working model of hexameric MurG on the membrane. (a) Tentative membrane-docking regions on MurG. Membrane-anchoring regions were predicted by the MODA server. Two regions, colored blue, were analyzed as tentative membrane-docking regions. $(b)$ The location of the second region, formed by a small helix connected by a loop, on each subunit of hexameric MurG. (c) Tentative working model of hexameric MurG on the membrane. 
may help in the design of next-generation antibiotics targeting MurG.

\section{Acknowledgements}

We thank the staff of the 5C beamline at PAL (Pohang, Korea) for their assistance with data collection.

\section{Funding information}

This study was supported by the Basic Science Research Program of the National Research Foundation of Korea (NRF) of the Ministry of Education, Science and Technology (NRF-2017M3A9D8062960 and NRF-2021R1A2C3003331). This research was part of the project entitled 'Development of potential antibiotic compounds using polar organism resources (15250103, KOPRI Grant PM21030)', funded by the Ministry of Oceans and Fisheries, Korea.

\section{References}

Basavannacharya, C., Moody, P. R., Munshi, T., Cronin, N., Keep, N. H. \& Bhakta, S. (2010). Protein Cell, 1, 1011-1022.

Brink-van der Laan, E. van den, Boots, J. P., Spelbrink, R. E. J., Kool, G. M., Breukink, E., Killian, J. A. \& de Kruijff, B. (2003). J. Bacteriol. 185, 3773-3779.

Bupp, K. \& van Heijenoort, J. (1993). J. Bacteriol. 175, 1841-1843.

Burki, T. K. (2018). Lancet Respir. Med. 6, 668.

Chen, V. B., Arendall, W. B., Headd, J. J., Keedy, D. A., Immormino, R. M., Kapral, G. J., Murray, L. W., Richardson, J. S. \& Richardson, D. C. (2010). Acta Cryst. D66, 12-21.

Chung, B. C., Mashalidis, E. H., Tanino, T., Kim, M., Matsuda, A., Hong, J., Ichikawa, S. \& Lee, S. Y. (2016). Nature, 533, 557-560.

DeLano, W. L. \& Lam, J. W. (2005). Abstr. Pap. Am. Chem. Soc. 230, U1371-U1372.

Emsley, P., Lohkamp, B., Scott, W. G. \& Cowtan, K. (2010). Acta Cryst. D66, 486-501.

Ha, S., Chang, E., Lo, M.-C., Men, H., Park, P., Ge, M. \& Walker, S. (1999). J. Am. Chem. Soc. 121, 8415-8426.

Ha, S., Walker, D., Shi, Y. \& Walker, S. (2000). Protein Sci. 9, 10451052.

Holm, L. \& Sander, C. (1995). Trends Biochem. Sci. 20, 478-480.
Hu, Y., Helm, J. S., Chen, L., Ginsberg, C., Gross, B., Kraybill, B., Tiyanont, K., Fang, X., Wu, T. \& Walker, S. (2004). Chem. Biol. 11, 703-711.

Hu, Y. N., Chen, L., Ha, S., Gross, B., Falcone, B., Walker, D., Mokhtarzadeh, M. \& Walker, S. (2003). Proc. Natl Acad. Sci. USA, 100, 845-849.

Kouidmi, I., Levesque, R. C. \& Paradis-Bleau, C. (2014). Mol. Microbiol. 94, 242-253.

Krissinel, E. \& Henrick, K. (2007). J. Mol. Biol. 372, 774-797.

Kufareva, I., Lenoir, M., Dancea, F., Sridhar, P., Raush, E., Bissig, C., Gruenberg, J., Abagyan, R. \& Overduin, M. (2014). Biochem. Cell Biol. 92, 555-563.

Kumar, M. (2016). Infect. Control Hosp. Epidemiol. 37, 365-366.

Laddomada, F., Miyachiro, M. M., Jessop, M., Patin, D., Job, V., Mengin-Lecreulx, D., Le Roy, A., Ebel, C., Breyton, C., Gutsche, I. \& Dessen, A. (2019). Sci. Rep. 9, 4656.

Liebschner, D., Afonine, P. V., Baker, M. L., Bunkóczi, G., Chen, V. B., Croll, T. I., Hintze, B., Hung, L.-W., Jain, S., McCoy, A. J., Moriarty, N. W., Oeffner, R. D., Poon, B. K., Prisant, M. G., Read, R. J., Richardson, J. S., Richardson, D. C., Sammito, M. D., Sobolev, O. V., Stockwell, D. H., Terwilliger, T. C., Urzhumtsev, A. G., Videau, L. L., Williams, C. J. \& Adams, P. D. (2019). Acta Cryst. D75, 861-877.

Matteï, P. J., Neves, D. \& Dessen, A. (2010). Curr. Opin. Struct. Biol. 20, 749-755.

McCoy, A. J. (2007). Acta Cryst. D63, 32-41.

Men, H. B., Park, P., Ge, M. \& Walker, S. (1998). J. Am. Chem. Soc. 120, 2484-2485.

Miyachiro, M. M., Granato, D., Trindade, D. M., Ebel, C., Paes Leme, A. F. \& Dessen, A. (2019). Biochemistry, 58, 3314-3324.

Mohammadi, T., Karczmarek, A., Crouvoisier, M., Bouhss, A., Mengin-Lecreulx, D. \& den Blaauwen, T. (2007). Mol. Microbiol. 65, 1106-1121.

Otwinowski, Z. \& Minor, W. (1997). Methods Enzymol. 276, 307-326.

Rocha, J., Sarkis, J., Thomas, A., Pitou, L., Radzimanowski, J., Audry, M., Chazalet, V., de Sanctis, D., Palcic, M. M., Block, M. A., GirardEgrot, A., Maréchal, E. \& Breton, C. (2016). Plant J. 85, 622-633.

Sham, L. T., Butler, E. K., Lebar, M. D., Kahne, D., Bernhardt, T. G. \& Ruiz, N. (2014). Science, 345, 220-222.

Shi, J., Liu, C. L., Zhang, B., Guo, W. J., Zhu, J., Chang, C.-Y., Zhao, E. J., Jiao, R. H., Tan, R. X. \& Ge, H. M. (2019). Chem. Sci. 10, $4839-4846$.

Smith, C. A. (2006). J. Mol. Biol. 362, 640-655.

Vollmer, W. \& Bertsche, U. (2008). Biochim. Biophys. Acta, 1778, 1714-1734. 